\title{
СЛОЖНЫЙ ИЗОМОРФИЗМ В МИНЕРАЛАХ ГРУППЫ РИНКИТА
}

\section{Селиванова Е.А., Лялина Л.М., Савченко Е.Э., Зозуля Д.Р.}

Геологический институт КНЦ РАН, Anamumbl, selivanova@geoksc.apatity.ru

К настоящему моменту опубликованы данные по двум титаносиликатам группы ринкита Сахарйокского массива, гайниту-(Y) [4] и батиеваиту-(Y) [5]. Однако, в породах массива, а именно в породах пегматита присутствуют многочисленные разновидности этих минералов, в которых при сохранении того же структурного мотива химический состав значительно варьирует. Общими элементами структур этих минералов являются трёхслойные $H O H$-пакеты, состоящие из внутреннего $O$-слоя, образованного октаэдрически (как правило) координированными катионами $\mathrm{Ti}, \mathrm{Na}, \mathrm{Ca}$, Mn, ...(позиции M2, M4, M5 табл. 1), и двух внешних гетерополиэдрических $H$-слоёв, состоящих из диортогрупп $\mathrm{Si}_{2} \mathrm{O}_{7}$ и 6- (7-) координированных катионов Ca, $R E E, \mathrm{Y}, \mathrm{Zr}$ (позиции M1, M3 табл. 1). Группа ринкита определяется стехиометрическим отношением $\mathrm{Ti}(+\mathrm{Nb}+\mathrm{Zr})=1$ для $\left(\mathrm{Si}_{2} \mathrm{O}_{7}\right)_{2}$.

Таблица 1. Идеальная структурная формула для минералов группы ринкита согласно [7], обозначения позиций по [3].

\begin{tabular}{|c|c|c|c|c|c|c|}
\hline \multirow[t]{2}{*}{ минерал } & \multicolumn{6}{|c|}{ Идеальная структурная формула } \\
\hline & M3 M1 & M4 M2 & M5 & $\left(\mathrm{Si}_{2} \mathrm{O}_{7}\right)_{\mathrm{n}}$ & $\mathrm{X} 8$ & X9 \\
\hline Мозандрит & $\left(\mathrm{Ca}_{3} R E E\right)$ & {$\left[\left(\mathrm{H}_{2} \mathrm{O}\right)_{2} \mathrm{Ca}_{0.5} \square_{0.5}\right]$} & $\mathrm{Ti}$ & $\left(\mathrm{Si}_{2} \mathrm{O}_{7}\right)_{2}$ & $(\mathrm{OH})_{2}$ & $\left(\mathrm{H}_{2} \mathrm{O}\right)_{2}$ \\
\hline Ринкит & $\left(\mathrm{Ca}_{3} R E E\right)$ & $\mathrm{Na}(\mathrm{NaCa})$ & $\mathrm{Ti}$ & $\left(\mathrm{Si}_{2} \mathrm{O}_{7}\right)_{2}$ & $(\mathrm{OF})$ & $\mathrm{F}_{2}$ \\
\hline Накарениобсит-(Се) & $\left(\mathrm{Ca}_{3} R E E\right)$ & $\mathrm{Na}_{3}$ & $\mathrm{Nb}$ & $\left(\mathrm{Si}_{2} \mathrm{O}_{7}\right)_{2}$ & (OF) & $\mathrm{F}_{2}$ \\
\hline Сейдозерит & $\mathrm{Na}_{2} \mathrm{Zr}_{2}$ & $\mathrm{Na}_{2} \mathrm{Mn}$ & $\mathrm{Ti}$ & $\left(\mathrm{Si}_{2} \mathrm{O}_{7}\right)_{2}$ & $\mathrm{O}_{2}$ & $\mathrm{~F}_{2}$ \\
\hline Гренмарит & $\mathrm{Na}_{2} \mathrm{Zr}_{2}$ & $\mathrm{Na}_{2} \mathrm{Mn}$ & $\mathrm{Zr}$ & $\left(\mathrm{Si}_{2} \mathrm{O}_{7}\right)_{2}$ & $\mathrm{O}_{2}$ & $\mathrm{~F}_{2}$ \\
\hline Розенбушит & $\mathrm{Ca}_{4} \mathrm{Ca}_{2} \mathrm{Zr}_{2}$ & $\mathrm{Na}_{2} \mathrm{Na}_{4}$ & TiZr & $\left(\mathrm{Si}_{2} \mathrm{O}_{7}\right)_{4}$ & $\mathrm{O}_{2} \mathrm{~F}_{2}$ & $\mathrm{~F}_{4}$ \\
\hline Кохит & $\mathrm{Ca}_{2} \mathrm{MnZr}$ & $\mathrm{Na}_{3}$ & $\mathrm{Ti}$ & $\left(\mathrm{Si}_{2} \mathrm{O}_{7}\right)_{2}$ & OF & $\mathrm{F}_{2}$ \\
\hline Гетценит & $\mathrm{Ca}_{2} \mathrm{Ca}_{2}$ & $\mathrm{NaCa}_{2}$ & $\mathrm{Ti}$ & $\left(\mathrm{Si}_{2} \mathrm{O}_{7}\right)_{2}$ & (OF) & $\mathrm{F}_{2}$ \\
\hline Гайнит-(Y) & {$\left[\mathrm{Ca}_{3}(\mathrm{Y}, R E E)\right]$} & $\mathrm{Na}(\mathrm{NaCa})$ & $\mathrm{Ti}$ & $\left(\mathrm{Si}_{2} \mathrm{O}_{7}\right)_{2}$ & (OF) & $\mathrm{F}_{2}$ \\
\hline Фогоит-(Y) & $\mathrm{Ca}_{2} \mathrm{Y}_{2}$ & $\mathrm{Na}_{3}$ & $\mathrm{Ti}$ & $\left(\mathrm{Si}_{2} \mathrm{O}_{7}\right)_{2}$ & (OF) & $\mathrm{F}_{2}$ \\
\hline Батиеваит-(Y) & $\mathrm{Ca}_{2} \mathrm{Y}_{2}$ & {$\left[\square_{1.0}\left(\mathrm{H}_{2} \mathrm{O}\right)_{2}\right]$} & $\mathrm{Ti}$ & $\left(\mathrm{Si}_{2} \mathrm{O}_{7}\right)_{2}$ & $(\mathrm{OH})_{2}$ & $\left(\mathrm{H}_{2} \mathrm{O}\right)_{2}$ \\
\hline
\end{tabular}

Основные схемы изоморфизма для гайнита-(Y), напрямую вытекающие из формул минералов [2]:

1. гетценит - гайнит-(Y) $2 \mathrm{Ca}^{2+} \leftrightarrow \mathrm{Y}^{3+}+\mathrm{Na}^{+}$

2. гайнит-(Y) - батиеваит-(Y): $2\left(\mathrm{Ca}^{2+}+\mathrm{Na}^{+}\right) \leftrightarrow \mathrm{Y}^{3+}+2 \mathrm{H}_{2} \mathrm{O}+\square$

характеризуются противонаправленным поведением натрия. Гайнит-(Y) - элемент как минимум двух различных рядов, причем для образцов из Сахарйока преобладает схема 2.

Задача анализа составов титаносиликатов также возникает из-за неразрешенной проблемы отношений двух минеральных фаз. Многочисленные совместные выделения минералов показывают [4-6] (рис. 1), что батиеваит-(Y), довольно неоднородный минерал с более рыхлой структурой, оказывается в центральной части зерна, в то время как каймы представлены прозрачным хорошо раскристаллизованным гайнитом-(Y). Обнаружены также подобные зерна, в центре которых находится не батиеваит-(Y), а фаза промежуточного состава.

Сложность структуры и вариации состава определяют применение метода главных компонент (МГК) для исследования корреляций элементов [1], в том числе элементов с малыми концентрациями. Статистическая обработка данных составов МГК позволит установить основные изоморфные замещения и выявить причины (факторы), которые определяют изменчивость и контролируют отношения минералов группы ринкита.

Исходя из особенностей строения, исследованные титаносиликаты были разделены на типы вещества со следующими характеристиками:

1. «Пористые» титаносиликаты - неоднородное вещество с многочисленными точечными де- 

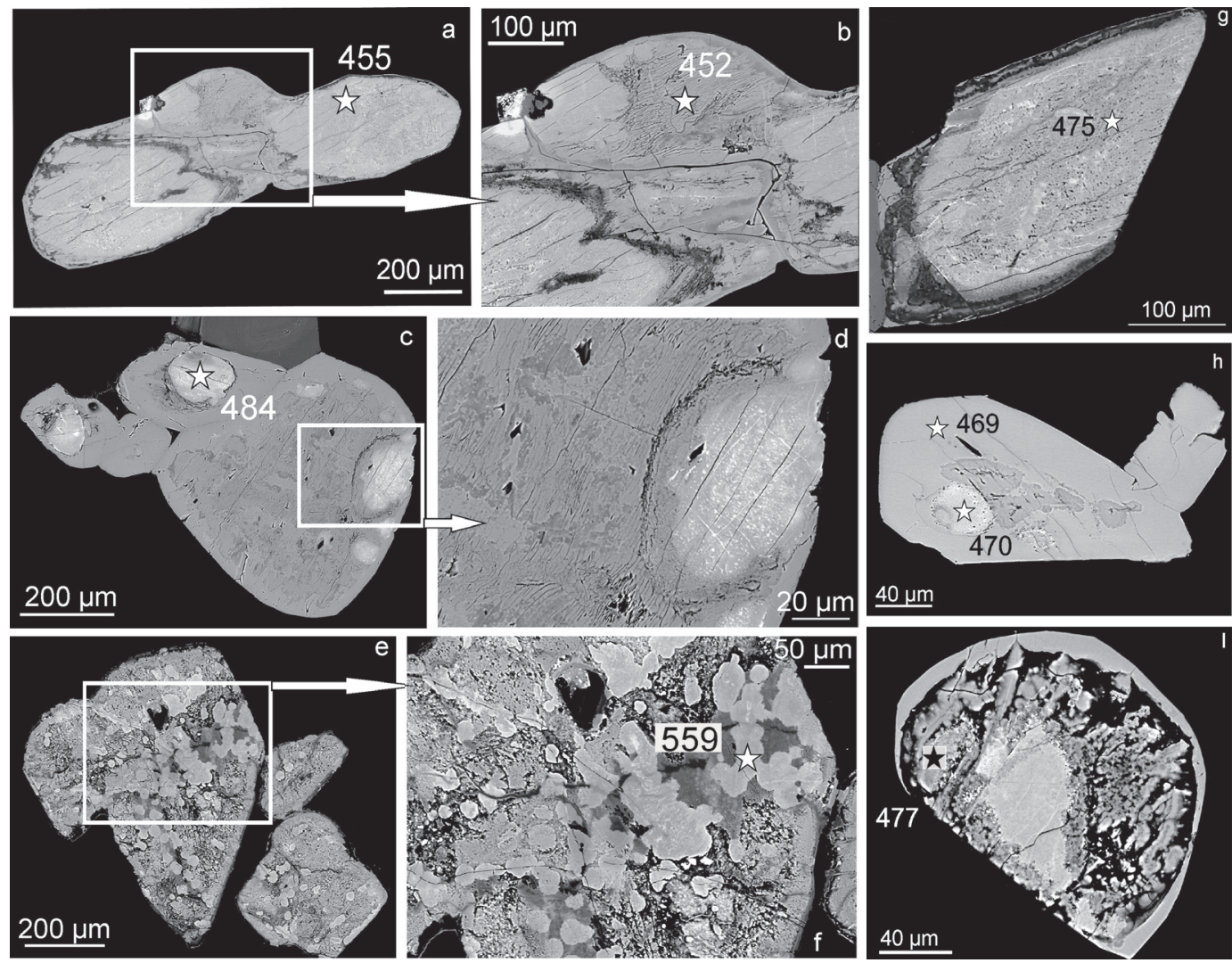

Рис. 1. Морфотипы минералов серии гайнит-(Y) - батиеваит-(Y), звезды - точки анализа.

фектами и трещинками, последние могут быть заполнены «тяжелым» светлым в BSE материалом, обогащенным титаном, иттрием, цирконием, ниобием, редкоземельными элементами (рис. $1 \mathrm{~g})=$ точки 6 на рис. 2.

2. «Слоистые» титаносиликаты имеют слюдоподобное строение, образованы многочисленными тонкими параллельными слоями (рис. $1 \mathrm{~b}$ ) = точки 4 на рис. 2.

3. «Глазки» - обособленные участки округлой, реже неправильной формы, располагающиеся внутри «слоистых» титаносиликатов. Их строение подобно «пористым» фазам (рис. $1 \mathrm{c}, \mathrm{d}, \mathrm{h})=$ точки 2 на рис. 2.

4. «Ровные» представляют собой участки округлой формы («монетки») отдельные или сливающиеся по несколько друг с другом в более обширные площади. Располагаются среди «рыхлых» или «пористых» титаносиликатов (рис. $1 \mathrm{e}, \mathrm{f}$ ) = точки 3 на рис. 2.

5. «Рыхлые» титаносиликаты представляют собой очень трещиноватое, хрупкое вещество, плохо поддающееся полировке (рис. 1 i) = точки 5 на рис. 2.

6. Гайнит-(Y) - образует самостоятельные идиоморфные призматические кристаллы или ксеноморфные зерна, но чаще внешние каймы обрастания вокруг титаносиликатов промежуточных составов (рис. 1h,i) = точки 1 на рис. 2 .

Опубликованные данные по батиеваиту-(Y) соответствуют нескольким слоистым и нескольким пористым разновидностям.

\section{Химический состав}

В работе использованы данные 79 точек анализа для образцов гайнита-(Y), батиеваита$(\mathrm{Y})$ и промежуточного состава фаз различной морфологии. В работе использованы данные 79 точек анализа для образцов гайнита-(Y), батиеваита-(Y) и промежуточного состава фаз различной морфологии. Химические анализы были выполнены на рентгеноспектральном микроанализаторе MS-46CAMECA, оснащённом волнодисперсионными спектрометрами,приускоряющемнапряжении 
22 кВ, токе электронного зонда 20-30 нА, диаметр пучка составлял 5-20 мкм. Применялись следующие образцы сравнения (аналитические линии): волластонит ( $\mathrm{SiK} \alpha, \mathrm{CaK} \alpha), \mathrm{Y}_{3} \mathrm{Al}_{5} \mathrm{O}_{12}(\mathrm{AlK} \alpha, \mathrm{YL} \alpha)$, лоренценит ( $\mathrm{TiK} \alpha, \mathrm{NaK} \alpha), \mathrm{ZrSiO}_{4}(\mathrm{ZrL} \alpha), \mathrm{Nb}(\mathrm{NbK} \alpha), \mathrm{MnCO}_{3}(\mathrm{MnK} \alpha)$, форстерит $(\mathrm{MgK} \alpha)$, гематит (FeK $\alpha)$, вадеит $(\mathrm{KK} \alpha), \mathrm{LaCeS}_{2}(\mathrm{LaL} \alpha), \mathrm{CeS}(\mathrm{CeL} \alpha), \mathrm{LiNd}\left(\mathrm{MoO}_{4}\right)_{2}(\mathrm{NdL} \alpha), \mathrm{GdS}(\mathrm{GdL} \alpha), \mathrm{Dy}_{3} \mathrm{Al}_{5} \mathrm{O}_{12}$ $(\mathrm{DyL} \alpha), \mathrm{ErPO}_{4}(\mathrm{ErL} \alpha), \mathrm{Tm}_{3} \mathrm{Al}_{5} \mathrm{O}_{12}(\mathrm{TmL} \alpha), \mathrm{Yb}_{3} \mathrm{Al}_{5} \mathrm{O}_{12}(\mathrm{YbL} \alpha), \mathrm{Y}_{2.8} \mathrm{Lu}_{0.2} \mathrm{Al}_{5} \mathrm{O}_{12}(\mathrm{LuL} \alpha)$, атакамит (ClK $\left.\alpha\right)$. Содержание $\mathrm{F}$ определялось с помощью энергодисперсионного спектрометра XFlash-5010 Bruker $\mathrm{Nano} \mathrm{GmbH}$, установленном на сканирующем электронном микроскопе LEO-1450. Параметры элктронного зонда: ускоряющее напряжение 20 кВ, ток зонда 0,5 нА. Время накопления спектра 200 сек. При расчёте использовался бесстандартный P/B-ZAF метод системы QuanTax-200.

Метод главных компонент, задействованный при исследовании, применялся с использованием программы статистической обработки данных STATISTICA 12.0.

В качестве переменных взяты формульные коэффициенты, полученные из расчета $\mathrm{Si}+\mathrm{Al}=4$. Применена процедура варимакс, главные компоненты выделены по критерию Кайзера.

\section{Результаты и обсуждение}

Согласно критерию Кайзера если фактор не выделяет дисперсию, эквивалентную, по крайней мере, дисперсии одной переменной $(\lambda j<1.0)$, то он исключается из рассмотрения. Данному условию удовлетворяют четыре фактора.

1. Проведенный анализ матрицы факторных нагрузок (табл. 1, рис.2а) показал, что первый фактор F1 (27\% дисперсии) связывает группу переменных (Ca, Na, F) тесной положительной корреляцией. График счетов (рис. 2 c) показывает, что F1 четко отделяет полнокатионные составы гайнита-(Y) (точки 1) от декатионизированных образцов (точки 2-6). В правой части графика (рис. 2 a, III квадрант) находится группа (Y, Mg, Р), отрицательно связанная с первой группой переменных, что отражает связь между декатионизацией с одной стороны и завышенным содержанием $\mathrm{Y}$ и наличием примесных элементов $(\mathrm{Mg}, \mathrm{P})$ с другой: $\mathrm{Ca}^{2+}+\mathrm{Na}^{+}+\mathrm{F}^{-} \rightarrow \square+\mathrm{Y}^{3+}+(\mathrm{OH})^{-}$и $\mathrm{Ca}^{2+}+\mathrm{Na}^{+}$

Таблица 2. Результаты факторного анализа состава минералов серии гайнит-(Ү) - батиеваит-(Ү).

\begin{tabular}{|c|c|c|c|c|}
\hline \multirow{2}{*}{ переменные } & \multicolumn{4}{|c|}{ Собственные векторы } \\
\hline & F 1 & F 2 & F 3 & F 4 \\
\hline$R E E$ & 0.756320 & 0.476891 & 0.041060 & -0.082863 \\
\hline $\mathrm{Y}$ & 0.754054 & -0.058515 & 0.394748 & 0.054088 \\
\hline $\mathrm{Na}$ & -0.624196 & -0.337260 & -0.420135 & -0.412386 \\
\hline $\mathrm{K}$ & 0.029323 & 0.141516 & 0.164027 & 0.802367 \\
\hline $\mathrm{Mg}$ & 0.822040 & -0.288034 & 0.016398 & 0.091280 \\
\hline $\mathrm{Ca}$ & -0.734975 & -0.183786 & 0.079822 & -0.440946 \\
\hline $\mathrm{Ti}$ & 0.038583 & 0.595748 & 0.154522 & 0.348134 \\
\hline $\mathrm{Zr}$ & 0.007376 & 0.643992 & 0.115776 & 0.387322 \\
\hline $\mathrm{P}$ & 0.709521 & -0.378585 & -0.111755 & -0.071313 \\
\hline $\mathrm{Nb}$ & -0.074589 & 0.843153 & 0.008262 & -0.076845 \\
\hline $\mathrm{Mn}$ & 0.145517 & 0.123806 & 0.734688 & 0.044299 \\
\hline $\mathrm{Fe}$ & -0.150331 & -0.055727 & 0.037116 & -0.700456 \\
\hline $\mathrm{F}$ & -0.715492 & -0.339113 & -0.316716 & -0.439336 \\
\hline $\mathrm{Cl}$ & -0.012788 & 0.038252 & 0.883334 & 0.069680 \\
\hline $\begin{array}{c}\text { Соб̈ственные } \\
\text { числа }\end{array}$ & 3.813428 & 2.240130 & 1.839141 & 1.999090 \\
\hline $\begin{array}{c}\text { Вклад в } \\
\text { дисперсию }\end{array}$ & 0.272388 & 0.160009 & 0.131367 & 0.142792 \\
\hline
\end{tabular}




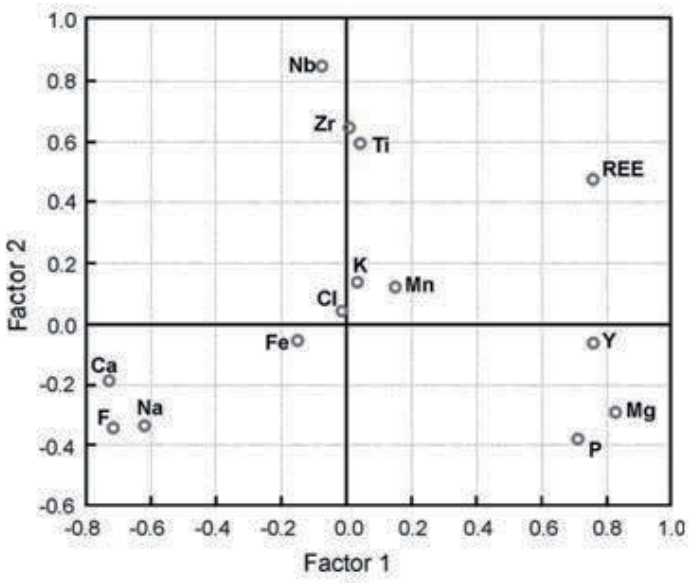

a

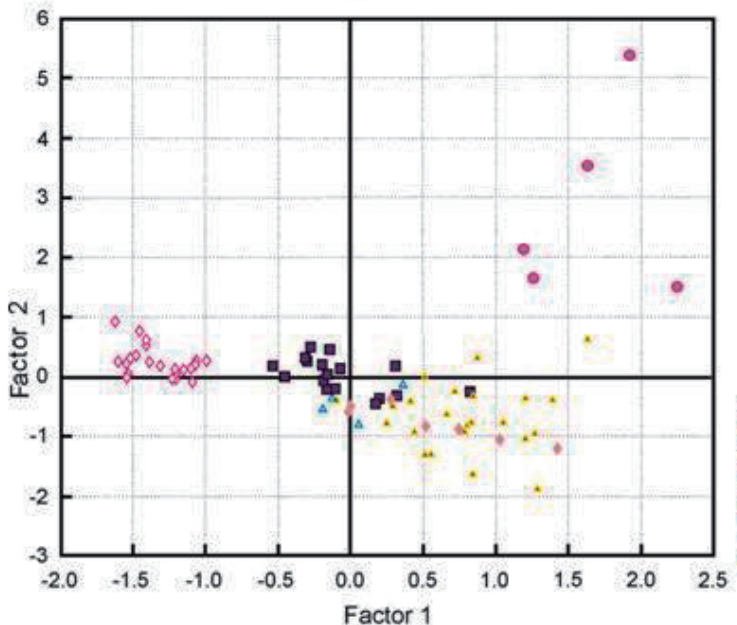

c

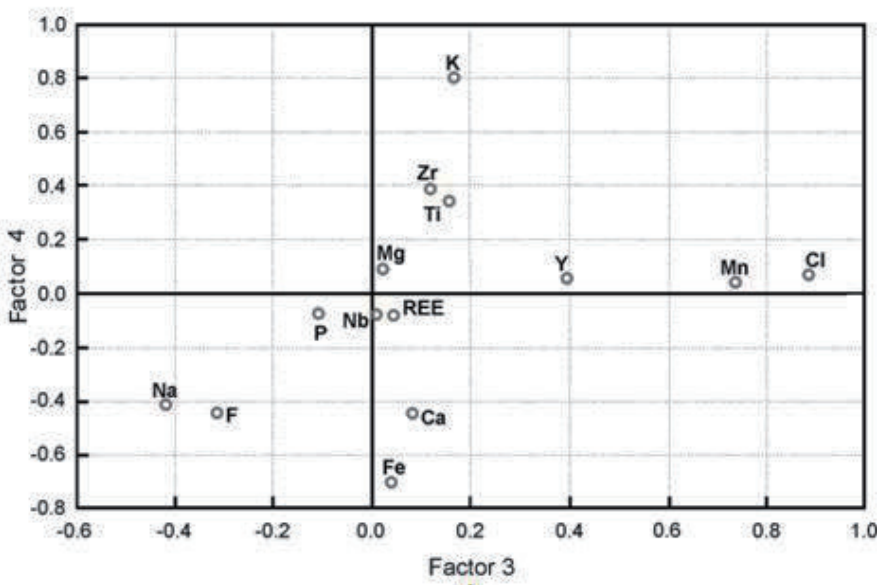

b

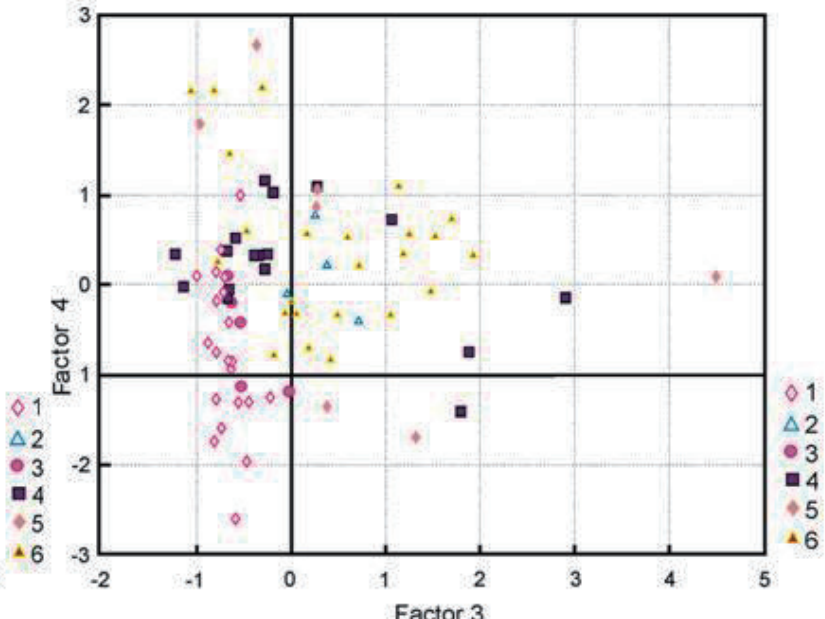

d

Рис. 2. Графики нагрузок $(\mathrm{a}, \mathrm{b})$ и графики счетов $(\mathrm{c}, \mathrm{d})$ по результатам факторного анализа. Обозначения к рис. 2 c, d приведены в тексте.

$+\mathrm{Si}^{4+} \rightarrow \square+\mathrm{Mg}^{2+}+\mathrm{P}^{5+}$. Не слишком отчетливо, но заметно разделение групп слоистых (точки 4) и пористых (точки 6).

Чем больше содержание в материале $\mathrm{Y}, \mathrm{Mg}, \mathrm{P}$, тем быстрее он разрушается, превращаясь в слоистое, либо в пористое вещество. Это могут быть как небольшие «глазки» (точки 2), так и почти полностью измененные кристаллы с узкой каймой гайнита-(Y) (точки 3-6).

2. Переменная REE имеет большие нагрузки как на F1, так и на F2 (рис.2а, II квадрант), она находится напротив группы (Ca, $\mathrm{Na}, \mathrm{F})$, формируя диагональ взаимоотношений между объектами, на графике счетов (рис. 2 с) с этим элементом специализирована группа ровные (точки 3). То есть участки с высокими $R E E$ также подвержены изменению, как и высокоиттриевые, но без деструкции, сохраняясь в виде реликтов. Различие маркируется схемой: $\mathrm{Mg}^{2+}+\mathrm{P}^{5+} \rightarrow R E E^{3+}+\mathrm{Si}^{4+}$.

Наибольшую нагрузку на вторую компоненту F2 (табл. 1, рис. 2 a) дает $\mathrm{Nb}$, связанный с Zr и $\mathrm{Ti}$, что отражает кристаллохимическое сродство элементов при отсутствии конкуренции в позиции M5 структуры. В случае гайнита-(Y) М5 может быть недозаполнена этими элементами, а в случае высокоиттриевых и особенно высокоредкоземельных точек сумма титана ниобия и циркония может доходить до 1.5 (нестехиометричный состав).

3. Третий фактор F3 (табл. 1, рис. 2 b) с большими нагрузками $\mathrm{Mn}$ и $\mathrm{Cl}$ говорит о том, что декатионизированные образцы, преимущественно пористые (точки 6) специализированы с $\mathrm{Mn}$ и $\mathrm{Cl}$. Группа ( $\mathrm{Na}, \mathrm{F})$ в отличие от группы $(\mathrm{Ca}, \mathrm{Na}, \mathrm{F})$ с нагрузкой на $\mathrm{F} 1$ дает нагрузки как на F3, так и на F4 (рис. 2 d, IV квадрант), соответствуя группе составов гайнита-(Y) на графике счетов (рис. 2 d). Это значит, что в данном случае можно говорить о специализации в отношении отдельных позиций 
структуры: (Na, F) соответствует позициям в O-слое, a (Ca, Na, F) - всем катионным позициям суммарно. Тогда лежащая напротив ( $\mathrm{Na}, \mathrm{F})$ во II квадранте группа ( $\mathrm{Zr}, \mathrm{Ti})$ легко объясняет, где находятся избыточный титан и цирконий - конечно в других позициях О-слоя, и чем больше таких смешанных позиций, тем менее устойчива структура к изменению.

4. Четвертым фактором F4 (табл. 1, рис. 2 b) с вкладом в дисперсию $14 \%$ дается простая схема изоморфных замещений $\mathrm{Ca}^{2+}+\mathrm{Fe}^{3+} \rightarrow \mathrm{K}^{+}+\mathrm{Ti}^{4+}$. Она работает как в отношении гайнита-(Y) (точки 1, выстроились вдоль F4), но также и для ровных (точки 3), высокоредкоземельных, и отвечает за устойчивость к деструктивному изменению. Схема не связана с выщелачиванием.

Работа поддержана грантом РФФИ № 16-05-00427 и в рамках госзадания № 0231-2015-0003.

\section{Литература}

1. ̆ёреског К.Г., Клован Д.И., Рейтент Р.А. Геологический факторный анализ. М.: Недра. 1980. 223 с.

2. Селиванова Е.А., Лялина Л.М. Минералы групп ринкита и розенбушита на Кольском полуострове // Сб. матер. XIII Всероссийской (с международным участием) Ферсмановской научной сессии «Региональная геология, минералогия и полезные ископаемые Кольского полуострова». ГИ КНЦ РАН. Апатиты, 4-5 апреля. 2016. С. 296-300.

3. Christiansen, C.C., Johnsen, O., Makovicky, E. Crystal chemistry of the rosenbuschite group // Canadian Mineralogist. 2003. V. 41. P. 1203-1224.

4. Lyalina L.M., Zolotarev Jr A.A., Selivanova E.A., Savchenko Ye.E., Zozulya D.R., Krivovichev S.V., Mikhailova Yu.A. Structural characterization and composition of Y-rich hainite from Sakharjok nepheline syenite pegmatite (Kola Peninsula, Russia) // Mineralogy and Petrology. 2015. V. 109. N 4. 443-451 p.

5. Lyalina L.M., Zolotarev A.A. Jr., Selivanova E.A., Savchenko Ye.E., Krivovichev S.V., Mikhailova Yu.A., Kadyrova G.I., Zozulya D.R. Batievaite-(Y), $\mathrm{Y}_{2} \mathrm{Ca}_{2} \mathrm{Ti}_{2}\left[\mathrm{Si}_{2} \mathrm{O}_{7}\right]_{2}(\mathrm{OH})_{2}\left(\mathrm{H}_{2} \mathrm{O}\right)_{4}$, a new mineral from nepheline syenite pegmatite in the Sakharjok massif, Kola Peninsula, Russia // Mineralogy and Petrology. 2016. V. 110(6). 895-904 p.

6. Lyalina, L., Zozulya, D., Selivanova, E., Savchenko Ye. Genetic relationship between batievaite-(Y) and hainite-(Y) from Sakharjok nepheline syenite pegmatite, Keivy alkaline province, NW Russia. Conference on accessory minerals, 13-17.09.2017. Vienna.

7. Sokolova E., Camara F. The seidozerite supergroup of TS-block minerals: nomenclature and classification, with change of the following names: rinkite to rinkite-(Ce), mosandrite to mosandrite-(Ce), hainite to hainite(Y) and innelite-1T to innelite-1A // Mineralogical Magazine. 2017 81. 1457-1484 p. 